\section{Aprendizaje de la organografía microscópica utilizando el método didáctico de la informática virtual en estudiantes universitarios}

\author{
Learning microscopic organography using the didactic method \\ of virtual computing in university students
}

\begin{abstract}
Resumen
El objetivo del estudio fue comparar el aprendizaje en la práctica guiada de la asignatura de Embriología e Histología Humana en alumnos de Odontología de dos universidades peruanas empleando dos técnicas de enseñanza de la organografía microscópica. Metodología: El estudio fue cuasi-experimental. Hubo un grupo control (50 estudiantes) en el que se utilizó la técnica tradicional y un grupo experimental (50 estudiantes) en el que se empleó la técnica virtual. En el grupo control se empleó un microscopio electrónico Zeiss para realizar las observaciones de las láminas mientras que en el grupo experimental se empleó un proyector multimedia. Se realizaron dos evaluaciones (con 20 preguntas cada una) para medir el aprendizaje sobre Embriología Estomatológica y sobre Histología Estomatológica, se obtuvo una nota por cada unidad y luego el promedio de ambas para obtener el aprendizaje global de la asignatura. Para comparar el aprendizaje entre los grupos se aplicó la prueba U de Mann Whitney. Resultados y conclusiones: El aprendizaje de los estudiantes acerca de la Embriología Estomatológica, Histología Estomatológica y global tuvo una calificación mediana \pm rango intercuartílico de $9 \pm 3,25,11 \pm 4$ y $10 \pm 2$ puntos respectivamente en el grupo control y de $11 \pm 2,25,13 \pm 2$ y $12 \pm 2$ puntos respectivamente en el grupo experimental. Al comparar el aprendizaje en las tres dimensiones se encontró diferencias estadísticamente significativas $(p<0,001)$. El aprendizaje global de la asignatura de Embriología e Histología Humana fue mayor en los alumnos que recibieron la enseńanza con ayuda de la técnica virtual que aquellos que lo recibieron con ayuda de la técnica tradicional.

Palabras clave: Educación en Odontología; Aprendizaje; Histología; Embriología; Evaluación Educacional.
\end{abstract}

\section{Abstract}

The aim of the study was to compare learning, of guided practices of Human Histology and Embryology subject in dental students from two peruvian universities using two techniques of microscopic organography teaching practices. Methodology: The study was quasi-experimental. There was a control group (50 students) on the traditional technique and an experimental group (50 students) where the virtual technique was used.

In the control group a Zeiss electron microscope was used for observing laminas while in the experimental group a multimedia projector was used. Two evaluations were conducted (with 20 questions each) to measure learning about Stomatologic Embryology and Stomatologic Histology, a note for each unit was obtained and the mean for the overall learning of the subject. For comparison between groups the U Mann Whitney test was applied. Results and conclusions: The student learning about Stomatologic Embryology, Stomatologic Histology and the global had a median \pm interquartile range of $9 \pm 3.25,11_{ \pm} 4$ and $10 \pm 2$ points respectively in the control group and $11 \pm 2.25,13 \pm 2$ and $12 \pm 2$ points respectively in the experimental group. Comparing learning of showed statistically significant differences $(\mathrm{p}<0.001)$. Global learning of the subject of Human Histology and Embryology was higher in students who received instruction using the virtual technique than those using the traditional technique.

Keywords: Education Dental; Learning; Histology; Embryology; Educational measurement.

\section{Introducción}

Las tecnologías de la información y las comunicaciones se han convertido en elementos sustantivos inherentes al desarrollo de todas las esferas de la vida.

La enseñanza de la Embriología e Histología Humana, por su complejidad y extensión, es un reto constante para docentes y estudiantes, lo que ha impul- sado la creación de nuevas estrategias pedagógicas que faciliten su aprendizaje, entre ellas, los entornos virtuales. Para ello la enseñanza práctica se basa en el uso del microscopio y en la observación de un número considerable de preparaciones y fotos microscópicas. La comprensión de las imágenes requiere un elevado grado de dedicación del profesor, ya que el alumno debe
Justiniano Sotomayor-Camayo ${ }^{1}$, Eufemia Correa-Olaya², Manuel A. Mattos-Vela ${ }^{3}$, Manuel A. Mendoza-Segura ${ }^{4}$, Carlos Valdivieso-Montoya ${ }^{3}$

1. Departamento Académico de Ciencias Básicas. Facultad de Odontología de la Universidad Nacional Mayor de San Marcos, Perú.

2. Departamento Académico de Estomatología Pediátrica. Facultad de Odontología de la Universidad Nacional Mayor de San Marcos, Perú.

3. Facultad de Odontología de la Universidad Nacional Mayor de San Marcos, Perú.

4. Facultad de Medicina de la Universidad Nacional Federico Villarreal, Perú.

\section{Correspondencia:}

Dr. Justiniano Sotomayor Camayo

Facultad de Odontología, Universidad Nacional Mayor de San Marcos, Av. German Amézaga s/n, Lima 1. Perú.

Correo electrónico: justinianosc@hotmail.com

\section{Coautores:}

Correa-Olaya: femisabel@hotmail.com Mattos-Vela: manuel_mattos@yahoo.com Mendoza-Segura: drmendozas@yohoo.es Valdivieso-Montoya: clinicaeltrebol@yahoo.es

Fecha de recepción: 11-08-14

Fecha de aceptación: 04-12-14

aprender a ver, reconocer e interpretar estructuras que pueden presentarse con aspectos distintos (coloraciones, sentido de la sección, y otros). A pesar de la utilización de los tradicionales atlas histológicos, la habituación a la interpretación de las imágenes microscópicas no es fácil para los alumnos. Por una parte, el tiempo disponible en el laboratorio, así como en muchos ca- 
sos el número de microscopios por alumno, son limitados. Generalmente los textos disponibles solo cubren la histología humana y en menor medida la estomatológica. Además, la mayoría de estos materiales didácticos tienen un alto precio, dada la necesaria utilización de un número elevado de imágenes en color, que hace que los alumnos desistan de su adquisición y que este tipo de textos no se encuentre en las bibliotecas universitarias en un número suficiente ${ }^{1}$.

El docente universitario quiere que el cambio se materialice en una enseñanza de mayor calidad, en un aprendizaje que apueste por el desarrollo de competencias, para esto es necesario que crea en ello y que ponga los medios para hacerlo efectivo. En este sentido, la pieza fundamental de la innovación en la docencia universitaria supone hacerlo en función del aprendizaje. Nuestro trabajo profesional debe dirigirse en hacer todo lo que esté a nuestro alcance para facilitar el acceso intelectual de nuestros estudiantes a los contenidos y prácticas profesionales en una determinada formación profesional, implicando a los alumnos en la búsqueda y elaboración del conocimiento, mediante las estrategias y actividades apropiadas. En el conocimiento didáctico no solamente tenemos que saber el cómo motivar y dar una clase, cómo elaborar un material para colgar e interaccionar en internet o cómo evaluar, importa mucho el por qué y para qué, es decir, las finalidades, saber por qué lo hago, por qué está orientado a la aprehensión de determinado perfil profesional o la obtención de determinados objetivos formativos, eso es lo que justifica que lo hagamos de determinada manera, lo que estamos intentando es dar respuesta a los elementos mucho más profundos, de mayor nivel, que tienen que ver con la formación.

El protagonismo de los estudiantes lleva siempre a activar el desarrollo de las clases y promover en ellos, un estilo dinámico de aprendizaje, a la vez que contribuye para que el docente comparta reflexiones, decisiones, interrogantes y propuestas ${ }^{2}$.

La buena enseñanza se refleja en el rendimiento académico a través de la evaluación de los conocimientos adquiridos sobre un tema específico. La evaluación y diagnóstico del conocimiento adquirido, permite determinar si se han cumplido o no los objetivos iniciales del proceso de aprendizaje y clasificarlos de acuerdo al nivel adquirido, para poder realizar una retroalimentación de dicho proceso y lograr un grupo más homogéneo. ${ }^{3}$

La presente investigación, buscó abordar esta problemática comparando el aprendizaje de esta área en estudiantes de Odontología expuestos a dos técnicas diferentes de enseñanza de la organografía microscópica: la tradicional y la virtual.

\section{Materiales y métodos}

Se realizó un estudio cuasi-experimental. La muestra estuvo constituida por los estudiantes de pregrado de la carrera profesional de Odontología que cursaron las prácticas de la asignatura de Histología y Embriología Humana de dos universidades peruanas matriculados en el semestre académico 2009-II, distribuidos en grupo control: 50 estudiantes del II ciclo de la Facultad de Odontologia de la Universidad Inca Garcilaso de la Vega (UIGV) de la ciudad de Lima y en un grupo experimental: 50 estudiantes del IV ciclo de la Universidad Peruana los Andes (UPLA) de la ciudad de Huancayo. Se trató de un muestreo no probabilístico.

Los criterios de inclusión considerados fueron: alumnos matriculados en la asignatura de Embriología e Histología Humana y asistencia regular a la asignatura de al menos un $70 \%$ de las clases de práctica guiada. Los criterios de exclusión fueron: alumnos que hayan repetido la asignatura.

\section{Procedimientos y Técnicas.}

Se solicitó el permiso correspondiente a las instituciones donde se realizó la investigación: Facultad de Odontología del grupo control (asignatura de Embriología e Histología General y Aplicada) y la Facultad de Ciencias de la Salud, Carrera Profesional de Odontología del grupo experimental (asignatura de Embriología e Histología Oral).

En los estudiantes del grupo control, se utilizó la técnica tradicional (microscopía) que consistió en lo siguiente: la práctica fue eminentemente de microscopía, utilizando un microscopio óptico de luz incorporada marca Zeiss (Germany), la observación se realizó con los objetivos panorámico, 10X, y 40X, y el ocular de 10x, el profesor explicó en diapositivas las láminas a observar, los estudiantes en una práctica de cuatro horas académicas semanales ( $45 \mathrm{minu}-$ tos por hora), trataron de identificar las células, tejidos, estructuras y órganos señalados para la práctica, utilizando las láminas donde las muestras estuvieron coloreadas y montadas en láminas porta objetos, protegidos por laminillas cubre objetos, el estudiante graficó lo que observó en una guía de prácticas. Esta fue la metodología en cada práctica en el laboratorio de Embriología de la Facultad de Estomatología del grupo control.

No hubo forma de observar nuevamente las láminas, no hubo acceso a ellas, tampoco se disponían de microscopios, al final, luego de concluir la unidad respectiva, hubo un paso con microscopio (un ensayo para el examen) y repaso de las láminas observadas en la unidad respectiva. La siguiente clase fue el examen respectivo con microscopios.

Los estudiantes del grupo experimental utilizaron la técnica virtual, que consistió en lo siguiente: en la práctica el profesor dio la explicación de las láminas a estudiar, por medio de un proyector multimedia y una memoria USB donde están grabadas las láminas de la práctica, que son las mismas utilizadas en el grupo control, a los mismos aumentos y coloración respectiva, esa práctica fue realizada en el aula de clase, los materiales audio visuales fueron un proyector multimedia, computadora y puntero láser.

Las prácticas duraron cuatro horas académicas, donde los estudiantes preguntaron por cada célula, tejido, estructura y órgano a identificar, luego los estudiantes grabaron en sus respectivas memorias las imágenes para observarlos en sus hogares cuantas veces deseen: al finalizar la unidad respectiva, se tomó un paso (un ensayo para el examen) y luego el profesor y los estudiantes repasaron cada lámina dando énfasis en las debilidades encontradas en cada estudiante.

Para medir el impacto de ambos métodos pedagógicos, se adaptó el examen práctico, aplicado a ambos grupos. Las láminas fueron proyectadas en un ecran y cada pregunta se marcó con el puntero láser. El objetivo de la evaluación fue medir la dimensión conceptual de la temática de Embriología e Histología Estomatológica, terminología, estructuras, órganos y tejidos, tanto de la embriología e histología básica como clínica.

Los contenidos impartidos y las evaluaciones realizadas (cada ocho semanas) fueron los mismos para ambas universidades: La primera evaluación fue sobre Embriología Estomatológica y la segunda fue sobre Histología Estomatológica. Cada evaluación constó de 10 ítems, pero cada ítem se desdobló en $\mathrm{A}$ y B, sumando un total de 20 preguntas que hacen 20 puntos. No se realizó una evaluación al inicio del curso para no evidenciar las preguntas que se to- 
marían posteriormente y así participen en igualdad de condiciones que el resto de cursos de ciencias básicas. Los estudiantes dieron su consentimiento para participar del estudio.

Un solo profesor fue quien enseñó las prácticas y evaluó a los alumnos de ambos grupos, para lo cual fue cegado a la identidad de cada estudiante.

Los exámenes (preguntas y metodología fueron iguales para las dos universidades). La recolección se realizó en el laboratorio de estudios microscópicos contando con luz adecuada donde participó el estudiante.

\section{Análisis estadístico.}

El procesamiento y análisis de los datos se realizó por medio del programa estadístico SPSS versión 15. Se realizó un análisis descriptivo de los datos em- pleando la mediana como medida de tendencia central y el rango intercuartílico como medida de desviación para la variable dependiente en los grupos control y experimental, puesto que la distribución de los datos era asimétrica, lo cual fue evidenciado a través de la prueba de Kolmogorov Smirnov. Para comparar el aprendizaje entre los dos grupos se aplicó la prueba no paramétrica U de Mann Whitney, trabajando a un nivel de significancia de 5\%.

\section{Resultados}

La muestra final de estudio estuvo constituida en el grupo control por 50 individuos (19 hombres, 38\%) y en el grupo experimental por 50 individuos (24 hombres, 48\%).

En el grupo control, el aprendizaje de los estudiantes de Odontología acerca de la Embriología Estomatológica, Histología Estomatológica y global tuvo una calificación mediana \pm rango intercuartílico de $9 \pm 3,25,11 \pm 4$ y $10 \pm 2$ puntos respectivamente. En el grupo experimental, el aprendizaje de los estudiantes en las mismas tres dimensiones tuvo una calificación de $11 \pm 2,25,13 \pm 2$ y $12 \pm 2$ puntos respectivamente (Tabla 1 ).

Para comparar el aprendizaje de Embriología Estomatológica, Histología Estomatológica y global producido en ambos grupos se empleó la prueba no paramétrica U de Mann Whitney encontrándose una diferencia estadística altamente significativa $(\mathrm{p}<0,001)$ en los tres casos, siendo los rangos promedio mayores en el grupo experimental (62,31, 64,04 y 66,44 respectivamente) respecto al control $(38,69,36,96$ y 34,56 respectivamente) (Tablas $2,3,4)$.

Tabla 1. Aprendizaje de los alumnos de pregrado de Odontología integrantes del grupo control y experimental

\begin{tabular}{lllcccc}
\hline Grupo & Aprendizaje & $\mathbf{n}$ & Mínimo & Máximo & Mediana & $\begin{array}{c}\text { Rango } \\
\text { intercuartilico }\end{array}$ \\
\hline Control & Embriología Estomatológica & 50 & 3 & 13 & 9 & 3,25 \\
& Histología Estomatológica & 50 & 2 & 16 & 11 & 4 \\
& Global & 50 & 6 & 13 & 10 & 2 \\
\hline \multirow{3}{*}{ Experimental } & Embriología Estomatológica & 50 & 5 & 15 & 11 & 2,25 \\
& Histología Estomatológica & 50 & 8 & 16 & 13 & 2 \\
& Global & 50 & 7 & 15 & 12 & 2 \\
\hline
\end{tabular}

Tabla 2. Comparación del aprendizaje de Embriología Estomatológica

\begin{tabular}{lllll}
\hline Grupo & $\mathrm{n}$ & $\begin{array}{l}\text { Rango } \\
\text { promedio }\end{array}$ & $\mathrm{U}$ & Valor $\mathrm{p}$ \\
\hline Control & 50 & 38,69 & 659,5 & $<0,001$ \\
Experimental & 50 & 62,31 & & \\
\hline
\end{tabular}

Tabla 3. Comparación del aprendizaje de Histología Estomatológica

\begin{tabular}{lllll}
\hline Grupo & $\mathbf{n}$ & $\begin{array}{l}\text { Rango } \\
\text { promedio }\end{array}$ & U & Valor $\mathbf{p}$ \\
\hline Control & 50 & 36,96 & 573 & $<0,001$ \\
Experimental & 50 & 64,04 & & \\
\hline
\end{tabular}

Tabla 4. Comparación del aprendizaje global

\begin{tabular}{lllll}
\hline Grupo & $\mathbf{n}$ & $\begin{array}{l}\text { Rango } \\
\text { promedio }\end{array}$ & $\mathbf{U}$ & Valor $\mathbf{p}$ \\
\hline Control & 50 & 34,56 & 453 & $<0,001$ \\
Experimental & 50 & 66,44 & & \\
\hline
\end{tabular}

\section{Discusión}

Los grupos control y experimental considerados en este estudio fueron integrados por estudiantes de dos universidades particulares de Lima y Huancayo respectivamente debido a la accesibilidad que tenía el investigador principal a ambas casas de estudio por ser docente en ellas. Los dos grupos presentaron características semejantes en su constitución, tanto en el nivel socioeconómico (pertenecían a universidades privadas) como en el sexo $(\mathrm{p}=0,313)$, lo cual, permitió controlar estas variables intervinientes. Si bien las muestras son pequeñas y sin asignación aleatoria, sin embargo, permiten tener un primer acercamiento al problema estudiado aportando evidencia científica que permitirá posteriores estudios con muestras mayores o en grupos diferentes.

Para realizar el proceso de enseńanza aprendizaje existen múltiples estrategias pedagógicas ${ }^{4}$ entre ellas, el uso de las nuevas tecnologías de la información y comunicación se vienen empleando cada vez con más frecuencia. 
No se dispone todavía de evidencias concluyentes del potencial transformador de los entornos virtuales de enseñanza aprendizaje, por lo que se sugiere mantener una perspectiva crítica y analítica orientada a estudiar, analizar y comprender el potencial transformador de las TIC en el marco de los usos que profesores y estudiantes hacen de estas tecnologías tanto en los procesos de construcción guiada como de coconstrucción del conocimiento 5 .

Por ello, el presente estudio aborda este tema, buscando comparar el aprendizaje producto de dos técnicas de enseñanza de la organografía microscópica en estudiantes de Odontología. Se observó que el aprendizaje fue menor en dos puntos en el grupo control (expuesto a la técnica tradicional) respecto al grupo experimental (expuesto a la técnica informática virtual) en las tres dimensiones estudiadas: Embriología Estomatológica, Histología Estomatológica y global, lo cual coincide con los resultados hallados por Castro ${ }^{6}$, Orellana et al. ${ }^{7}$ y Quijano $^{8}$, quienes compararon un método tradicional de enseñanza frente a un método de autoaprendizaje con soporte virtual en estudiantes de odontología, a) un software RecomX para la enseńanza aprendizaje de contenidos conceptuales y procedimentales odontológicos y a un b) learning en estudiantes de medicina, respectivamente. Si bien, la metodología virtual y el tipo de evaluación empleados en cada estudio fueron algo distintos, sin embargo, ello no cambió la superioridad del método virtual, habiéndose encontrado una nota promedio de 14,5 (con 97,7\% de aprobados) frente a 9,5 (con $40 \%$ de aprobados), $18,1+1,8$ frente a $11,7+3,3$ y $37,8 \%$ de aprobados frente a 6,9\% en los grupos experimental y control respectivamente de cada estudio antes mencionado.

Es probable que la poca superioridad del método virtual encontrada en este estudio, solo dos puntos más que la mediana encontrada con el método tradicional, se haya debido al empleo del método virtual en su mínima expresión, es decir, solamente poniendo al alcance de los estudiantes las imágenes de los cortes histológicos para su revisión indefinida, mientras que en el estudio de Castro ${ }^{6}$ además del soporte virtual del método de autoaprendizaje se empleó también un tutor/evaluador virtual, mientras que en el estudio de Quijano $^{8}$ junto al b) learning se empleó también mapas conceptuales, guías de estudio e imágenes computarizadas de neuroanatomía montadas en la plataforma moodle, con guías interactivas, animaciones físicas de procedimientos, foro virtual y uso de chat.

Se requieren estudios que evalúen aún más el uso del método virtual empleando las diferentes herramientas que esta puede brindar en cursos de Histología y Embriología, lo cual podría incrementar aun más el aprendizaje de los estudiantes en estas materias. También se requieren estudios cualitativos que evalúen ambas técnicas de enseñanza de la organografía microscópica para complementar los resultados de los estudios cuantitativos.

Se debe considerar además, que estos métodos virtuales son mejor aceptados por los estudiantes según lo demostraron Guiraldes et al.9 quienes diseñaron, aplicaron y validaron nuevos métodos de enseńanza activa, interactiva y participativa mediante la participación de software al curso de Anatomía y Embriología Humana y encontraron que la opinión prevalente de los estudiantes fue que los programas computacionales constituyen importante apoyo para facilitar el aprendizaje. Por ello, el método virtual de enseñanza de la organografía microscópica muestra ventajas interesantes que deben ser consideradas para mejorar el aprendizaje de los estudiantes ${ }^{10}$.

Se recomienda realizar más investigaciones semejantes a la presente empleando otras muestras de estudiantes para verificar los resultados encontrados en este estudio, buscando aleatorizar la asignación de estudiantes a los grupos control y experimental, evaluar el aprendizaje post intervención en más periodos de tiempo, así como, controlar también otras posibles variables intervinientes.

\section{Conclusión}

Se concluye que los alumnos de pregrado de Odontología que recibieron la enseñanza de la organografía microscópica en la práctica guiada con ayuda de la técnica informática virtual tuvieron un mayor aprendizaje cognitivo de la Embriología Estomatológica, de la Histología Estomatológica y global que aquellos que la recibieron con ayuda de la técnica tradicional.

\section{Referencias bibliográficas}

1. Ávila R, Samar M, Peñaloza F. Creación de un laboratorio virtual para la enseñanza universitaria de la Embriología Humana en sus aspectos biológicos, éticos y sociales. Facultad de Ciencias Médicas. Universidad Nacional de Córdoba, Argentina. Int J Odontostomat. 2011;5(1):13-22.

2. Alonso C. Análisis y Diagnóstico de los Estilos de Aprendizaje de los Universitarios. Madrid: Universidad Complutense; 1991.

3. Alonso C, Gallego D, Honey P. Los Estilos de Aprendizaje. Procedimientos de diagnóstico y Mejora. 3 ed. Bilbao: Ediciones Mensajero. 1994.

4. Videla RL. Clases pasivas, clases activas y clases virtuales ¿transmitir o construir conocimientos? RAR. 2010;74(2):187-191

5. Bustos A, Coll C. Los entornos virtuales como espacios de enseñanza y aprendizaje. Una perspectiva sicoeducativa para su caracterización y análisis. RMIE. 2010;15(44):163-184

6. Castro A. Autoaprendizaje con soporte virtual y éxito académico del estudiante de odontología de la Universidad Nacional Mayor de San Marcos. (Tesis doctoral). Perú: Universidad Nacional Mayor de San Marcos; 2007.

7. Orellana G, Morales A, García C, Ramírez R. La Hipermedia y la Enseńanza-Aprendizaje de la Odontología: Proyecto factible empleando el Software Recompx. Acta odontológica venezolana 2008;46(4):1-12.

8. Quijano Y. Impacto del uso de entornos virtuales de aprendizaje para la enseńanza de Neuroanatomía en estudiantes de Medicina. Universidad de Ciencias Aplicadas y Ambientales. 2010;13(2):15-22.

9. Guiraldes H, Oddó H, Mena B. Velasco N. Enseñanza de la Anatomía Humana: experiencias y desafíos en una escuela de medicina. Rev Chil Anat. 2001;19(2)

10. Salinas J, Aguaded J, Cabero J. Tecnologías para la educación. Diseño, producción y evaluación de medios para la formación docente. Madrid: Alianza Editorial; 2004. 